\title{
A New Method of Polyline Approximation
}

\author{
Alexander Gribov and Eugene Bodansky \\ Environmental System Research Institute (ESRI) \\ 380 New York St., Redlands, CA 92373-8100, USA \\ \{agribov, ebodansky@esri.com\}
}

\begin{abstract}
Many methods of a raw vectorization produce lines with redundant vertices. Therefore the results of vectorization usually need to be compressed. Approximating methods based on throwing out inessential vertices are widely disseminated. The result of using any of these methods is a polyline, the vertices of which are a subset of source polyline vertices. When the vertices of the source polyline contain noise, vertices of the result polyline will have the same noise. Reduction of vertices without noise filtering can disfigure the shape of the source polyline. We suggested a new optimal method of the piecewise linear approximation that produces noise filtering. Our method divides the source polyline into clusters and approximates each cluster with a straight line. Our optimal method of dividing polylines into clusters guarantees that the functional, which is the integral square error of approximation plus the penalty for each cluster, will be the minimum one.
\end{abstract}

Keywords: vectorization, polyline compression, polyline approximation, shape analysis.

\section{Introduction}

Many methods of a raw vectorization produce lines with redundant vertices. Therefore the results of vectorization usually need to be compressed. In additional to compression, noise filtering usually should be done, because a noise caused by source document distortion, scanning, and binarization may be too big.

Compression methods based on throwing out inessential vertices are widely disseminated, maybe because of their simplicity [1-5]. The result of using any of these methods is a polyline, the vertices of which are a subset of source polyline vertices. If the vertices of the source polyline contain noise, vertices of the result polyline obtained with compression will have the same noise. Reduction of vertices without noise filtering can disfigure the shape of the source polyline. So as we have mentioned before [6], compression methods have to be used cautiously.

Pavlidis and Horwitz [7] suggested a method of the piecewise linear approximation that produces not only compression, but also noise filtering. The source polyline is divided by some vertices that we call "critical points" into polygonal sectors or clusters. The method of least squares is later used for approximating clusters with straight lines. The main problem consists of finding the critical points.

The «split and merge» method suggested by Pavlidis and Horwitz defines the minimum number of critical points such that the maximum deviation of approximation of each cluster with the straight line or the sum of integral square errors of each cluster is not more than a given threshold. The algorithm is suboptimal. 
The vertices of the result polyline are intersections of adjacent approximating straight lines. These vertices do not necessary coincide with vertices of the source polyline. The threshold of approximation error controls the precision of approximation. The precision of the result polyline could be better than the precision of the noisy source line if a suitable threshold was selected.

Our algorithm divides the source polyline into clusters and approximates each cluster with a straight line similarly to the aforementioned algorithm. The technique of choosing critical points guarantees that the functional that is the integral square error of approximation plus the penalty for each critical point will be the minimum one.

\section{Problem Statement}

a. Let $p_{i}$, where $i=0, \ldots, n$, are vertices of the source polyline $P$. Let decomposition vertices $p_{q_{j}}$ divide $P$ into the set of non-overlapping polygonal sectors, where $q_{j},(j=0, \ldots m)$, are the indices of source polyline vertices (see Fig. 1). The number of decompositions of $P$ into $m$ sectors is $C_{n-1}^{m-1}$. The number of all possible decompositions of $P$ is $2^{n-1}$.

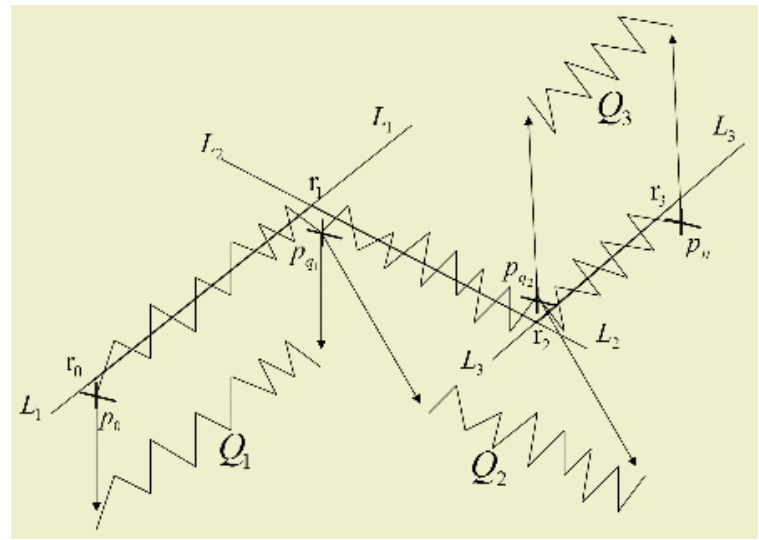

Fig. 1. Decomposition of the source polyline $P$ into polygonal sectors.

b. Approximates each polygonal sector $Q_{j}$ with a straight line $L_{j}$ minimizing integral square error $\varepsilon_{q_{j-1}, q_{j}}$ (see Appendix 1).

c. Let the measure of the error of the polyline approximation be a functional

$$
F(m,\{q\}, \Delta)=\sum_{j=1}^{m} \varepsilon_{q_{j-1}, q_{j}}+m \cdot \Delta,
$$

where $\Delta(\Delta \geq 0)$ - is a penalty for each straight segment of the result polyline. 
d. For a given $\Delta$, let the optimal decomposition be the decomposition for which functional $F$ is minimal. Approximation precision depends on the penalty $\Delta$. The smaller $\Delta$ is, the more decomposition points will be found. If $\Delta=0$, all vertices of the source polyline are decomposition points and $F=0$. As $\Delta \rightarrow \infty$, the source polyline becomes one polygonal sector.

e. After finding the optimal decomposition or critical points, polygonal sectors have to be approximated by optimal straight lines. Then, the intersections of the lines approximating the adjusted polygonal sectors should be found. The new polyline goes through these intersections $r_{j},(j=0, \ldots, m)$. The beginning and the end of the new polyline are built as projections of the beginning and the end points of the source polyline to the first and last straight segments of the new polyline.

\section{Iterative Algorithm for Obtaining an Optimal Solution}

Let $P_{i}, i \leq n$ be polylines defined by the first $i$ straight segments of the source polyline $P$.

Given that the optimal decompositions of $P_{k}, P_{k-1}, \ldots, P_{1}$ are known, we know the optimal (minimal) values of functional (1), the number of decomposition sectors $m_{i},(i=1, \ldots, k)$, and the decomposition points $q_{s}^{i},\left(i=1, \ldots, k ; s=1, \ldots, m_{i}\right)$ for each of these polylines.

Find the optimal decomposition of $P_{k+1}$. This task may be solved with an exhaustive search.

Let $M_{k+1}^{j}$ be a minimum value of the functional, when the last decomposition point of polyline $P_{k+1}$ is $p_{j}$. Obviously, $M_{k+1}^{j}=M_{j}+\varepsilon_{j, k+1}+\Delta$ and $M_{0}=0$.

Let $M_{k+1}^{j}$ be minimal when the value of $j,(j=k, k-1, \ldots, 0)$ is equal to $j^{*}$, or $M_{k+1}=M_{k+1}^{j^{*}}$.

The optimal decomposition of the source polyline will be built when $k=n-1$.

The computational complexity of this algorithm is $O\left(n^{2}\right)$, because the complexity of the calculation of $\varepsilon_{j, k+1}$ is $O(1)$ [7].

\section{Optimization}

The described algorithm can be accelerated by the minimum estimation $M_{k+1}^{j_{1}, j_{2}}$ of the functional $M_{k+1}^{j}$ for the case, when $j$ is located in the half-open interval $\left[j_{1}, j_{2}\right)$. If $M_{k+1}^{j_{3}}$ was found for some $j_{3}$ which does not belong to $\left[j_{1}, j_{2}\right)$, and $M_{k+1}^{j_{3}}$ is less 
than $M_{k+1}^{j_{1}, j_{2}}$, then the beginning of the last polygonal sector for optimal decomposition cannot belong to $\left[j_{1}, j_{2}\right)$ and it is not necessary to analyze vertices located inside this half-interval.

In Appendix 2 two minimum estimations were found

$$
S_{k+1}^{j_{1}, j_{2}}=M_{j_{1}}+\varepsilon_{j_{2}-1, k+1}+\Delta \text { and } K_{k+1}^{j_{1}, j_{2}}=M_{j_{2}-1}+\varepsilon_{j_{2}-1, k+1} .
$$

Therefore

$$
M_{k+1}^{j_{1}, j_{2}}=\max \left\{M_{j_{1}}+\Delta, M_{j_{2}-1}\right\}+\varepsilon_{j_{2}-1, k+1} .
$$

The minimum estimation (2) essentially accelerates the algorithm because the last point of the optimal decomposition of a long line is usually located closer to its end than to its beginning. The optimized algorithm is described in Appendix 3.

Fig. 2 compares the result of approximation obtained by suggested method and with a compression algorithm of Douglas-Peucker [1]. A regular decagon was used as ground-truth polyline. After densification, 1000 points were calculated. To obtain the source polyline, white noise was added to the coordinates of these points.

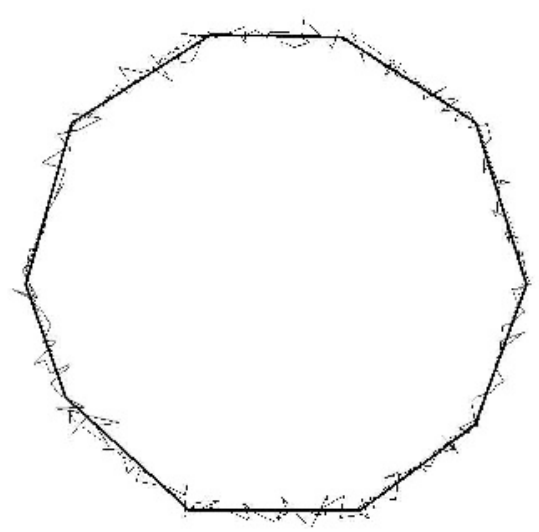

a)

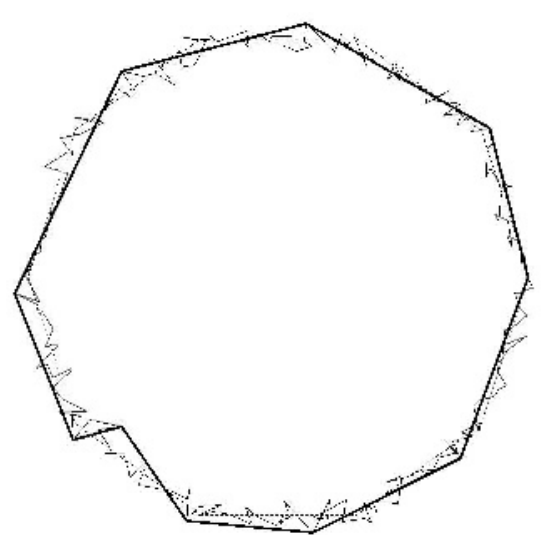

b)

.... The ground-truth decagon

- The source polyline (densified and noisy)

- The result of approximation

Fig. 2. The result of approximation of the regular decagon with a) the suggested method and b) Douglas-Peucker compression [1].

Fig. 3 shows the dependence of the optimal value of functional (1) on penalty $\Delta$, and Fig. 4 shows the dependence of the number of polygonal sectors on penalty $\Delta$ for the optimal decomposition of the source polyline. The horizontal segments in Fig. 4 show intervals of $\Delta$, inside which the optimal number of segments are constant and decomposition vertices are not changed. The interval of $\Delta$, corresponding to the result polyline with 10 segments, is the longest (in logarithmic scale) with the 


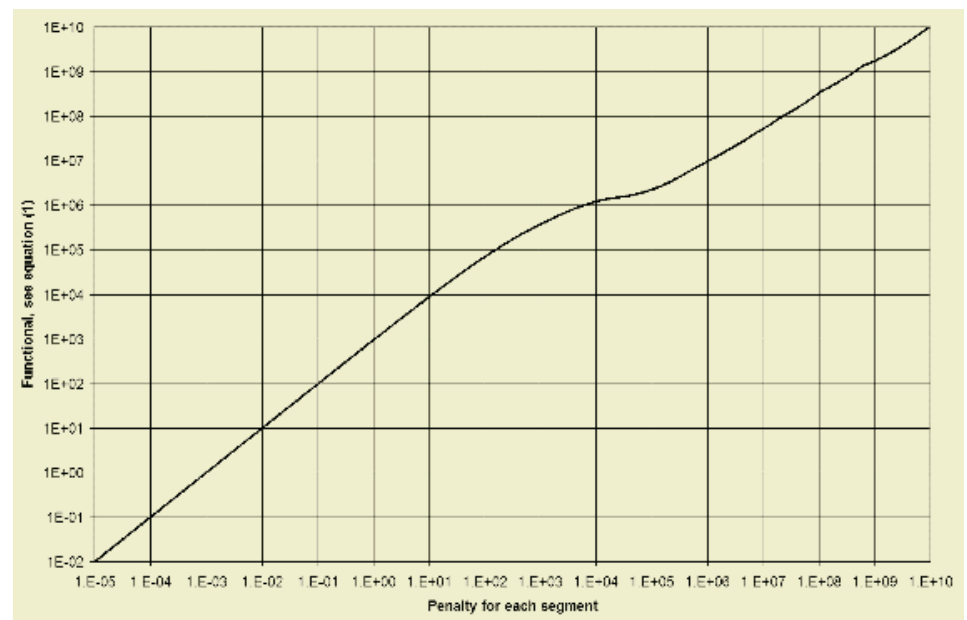

Fig. 3. Dependence a value of the functional (1) on the penalty $\Delta$.

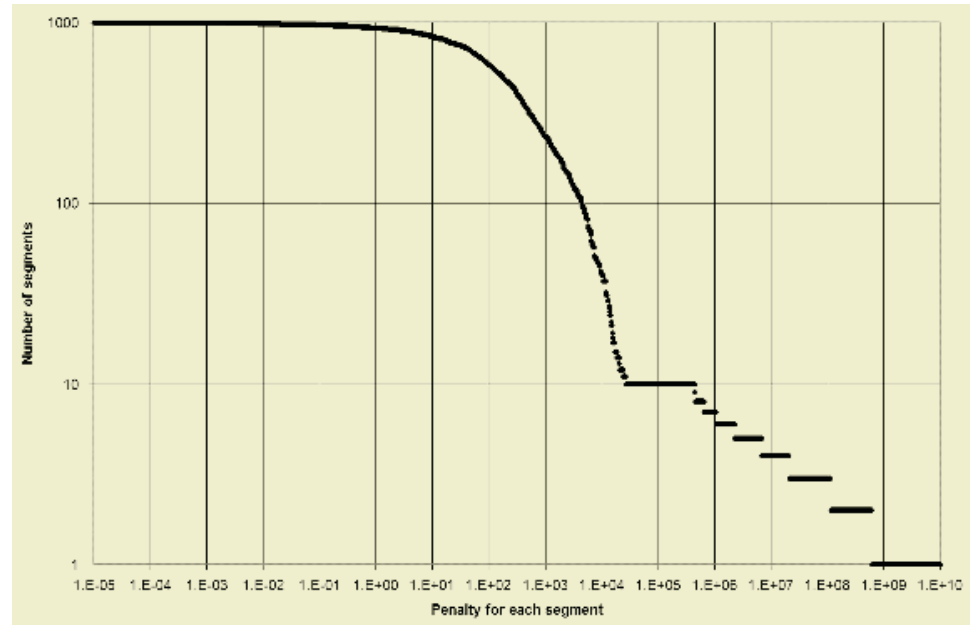

Fig. 4. Dependence the number of polygonal sectors on the penalty $\Delta$.

exception of a singular case (the end interval). It is evidence of the stability of the algorithm to variations of $\Delta$.

\section{Conclusion}

The goal of the article is to build an algorithm of filtering random errors of polylines. Suggested algorithm approximates the source noisy polyline with the new one. The vertices of the new polyline do not necessary coincide with vertices of the source polyline. The algorithm is based on the minimization of the functional that is the sum 
of the integral square error of approximation and a penalty $\Delta$ for each vertex of the result polyline.

Because the algorithm filters random noise the result polyline describes the shape of the ground-truth polyline better than the noisy source polyline. Therefore our algorithm is good for recognition of critical points.

The number of vertices of the result polyline usually is less than of the source polyline. Therefore the suggested algorithm can be used for compression. In the article we do not discuss the problem of stability of result polyline vertices found as intersections of straight lines. One more problem that was not analyzed here is selection of value of penalty $\Delta$. Meanwhile correctness of selection of $\Delta$ can be evaluated only by the operator.

\section{References}

1. D.Douglas, Th.Peucker, "Algorithm for the reduction of the number of points required to represent a digitized line or its caricature”, The Canadian Cartographer, Vol.10, \#2 (1973), pp.112-122.

2. T.Lang, "Rules for robot draftsmen", Geographical Magazine, V.22, N.1, (1969), pp.50-51.

3. U.Ramer, "Extraction of Line Structures from Photographs of Curved Objects", Computer Graphics and Image Processing, Vol.4 (1975), pp.81 - 103.

4. J.Sklansky, V.Gonzalez, "Fast Polygonal Approximation of Digitized Curves", Pattern Recognition, Vol.12 (1980), pp.327-331.

5. D.G.Lowe, "Perceptual organization and visual recognition", 1985, Ch. 4, Kluwer Academic Publishers, Boston.

6. E.Bodansky, A.Gribov, M.Pilouk, "Smoothing and Compression of Lines Obtained by Raster-to-Vector Conversion”, GREC 2002, LNCS 2390, D.Blostein, Y.-B.Kwon (Eds), 2002, pp.256-265.

7. Th.Pavlidis, S.L.Horwitz, "Segmentation of Plane Curves", IEEE Transactions on Computers, V.23, N.8, (1974), pp.860-870.

\section{Appendix 1}

The parametric description of the source line is $(x(t), y(t)), t \in[0, T]$ - where $(x(t), y(t))$ are the coordinates, $t$ is the distance between the beginning and the current point of the polyline measured along the polyline, $T$ is the polyline length.

Let $\varepsilon_{j_{1}, j_{2}}^{\alpha, C}=\int_{l_{j_{1}}}^{l_{j_{2}}}(x(t) \cos (\alpha)+y(t) \sin (\alpha)+C)^{2} d t$ be an integral square error of approximation of the polygonal sector, located between $j_{1}$-th and $j_{2}$-th vertices of the source polyline, with straight line $\cos (\alpha) \cdot x+\sin (\alpha) \cdot y+C=0$.

Let $\varepsilon_{j_{1}, j_{2}}$ be the minimum integral square error, i.e. $\varepsilon_{j_{1}, j_{2}}=\min _{\alpha, C}\left\{\varepsilon_{j_{1}, j_{2}}^{\alpha, C}\right\}$

The optimal approximating straight line is defined with the next $\cos (\alpha), \sin (\alpha)$, and $C$. 


$$
\begin{aligned}
& \cos (\alpha)=\left\{\begin{array}{l}
\frac{2 V_{x y}}{\sqrt{\omega^{2}+4 V_{x y}^{2}}}, V_{x x} \geq V_{y y} \\
-\frac{\omega}{\sqrt{\omega^{2}+4 V_{x y}^{2}}}, V_{x x}<V_{y y}
\end{array}\right. \\
& \sin (\alpha)=\left\{\begin{array}{l}
-\frac{\omega}{\sqrt{\omega^{2}+4 V_{x y}^{2}}}, V_{x x} \geq V_{y y} \\
\frac{2 V_{x y}}{\sqrt{\omega^{2}+4 V_{x y}^{2}}, V_{x x}<V_{y y}},
\end{array}\right. \\
& C=-\frac{V_{x} \cdot \cos (\alpha)+V_{y} \cdot \sin (\alpha)}{l_{j_{2}}-l_{j_{1}}},
\end{aligned}
$$

where $l_{j}$ is the distance between the beginning of the polyline and $j$-th vertex measured along the polyline,

$$
\begin{aligned}
& \omega=\sqrt{\left(V_{x x}-V_{y y}\right)^{2}+4 V_{x y}^{2}}+\left|V_{x x}-V_{y y}\right|, \\
& V_{x}=\int_{l_{j_{1}}}^{l_{j_{2}}} x(t) d t, V_{y}=\int_{l_{j_{1}}}^{l_{j_{2}}} y(t) d t, \\
& V_{x x}=M_{x x}-V_{x}^{2}, V_{x y}=M_{x y}-V_{x} \cdot V_{y}, V_{y y}=M_{y y}-V_{y}^{2}, \\
& M_{x x}=\int_{l_{j_{1}}}^{l_{j_{2}}} x^{2}(t) d t, M_{x y}=\int_{l_{j_{1}}}^{l_{j_{2}}} x(t) y(t) d t, M_{y y}=\int_{l_{j_{1}}} y^{2}(t) d t .
\end{aligned}
$$

The minimum integral square error equals [7]:

$$
\varepsilon_{j_{1}, j_{2}}=\frac{1}{2}\left(V_{x x}+V_{y y}-\sqrt{\left(V_{x x}-V_{y y}\right)^{2}+4 V_{x y}^{2}}\right) \text {. }
$$

\section{Appendix 2}

$$
\begin{aligned}
& \min _{j_{1} \leq j^{*}<j_{2}}\left\{M_{j^{*}}+\varepsilon_{j^{*}, k+1}+\Delta\right\}_{M_{j^{*}} \geq M_{j_{1}}, \varepsilon_{j^{*}, k+1} \geq \varepsilon_{j_{2}-1, k+1}} M_{j_{1}}+\varepsilon_{j_{2}-1, k+1}+\Delta, \\
& \min _{j_{1} \leq j^{*}<j_{2}}\left\{M_{j^{*}}+\varepsilon_{j^{*}, k+1}+\Delta\right\}_{\varepsilon_{j^{*}, k+1} \geq \varepsilon_{j^{*}, j_{2}-1}+\varepsilon_{j_{2}-1, k+1}} \\
& \geq \min _{j_{1} \leq j^{*}<j_{2}}\left\{M_{j^{*}}+\varepsilon_{j^{*}, j_{2}-1}+\varepsilon_{j_{2}-1, k+1}+\Delta\right\}_{M_{j^{*}}+\varepsilon_{j^{*}, j_{2}-1}} \geq \Delta \geq M_{j_{2}-1} M_{j_{2}-1}+\varepsilon_{j_{2}-1, k+1} .
\end{aligned}
$$


These inequalities can be proven with two obvious expressions:

$\varepsilon_{j_{1}, j_{2}}+\varepsilon_{j_{2}, j_{3}} \leq \varepsilon_{j_{1}, j_{3}} \forall 0 \leq j_{1} \leq j_{2} \leq j_{3} \leq n$ and $M_{j_{1}} \leq M_{j_{2}} \forall 0 \leq j_{1} \leq j_{2} \leq n$.

\section{Appendix 3: The Fast Algorithm of Optimal Decomposition of the Polyline $P_{k+1}$}

1. Calculates the functional $M_{k+1}^{\text {current }}=M_{k}+\varepsilon_{k, k+1}+\Delta=M_{k}+\Delta$ supposing that a new decomposition point is the vertex of the source polyline with index $j^{\text {current }}=k$.

2. Calculates with expression (2) the minimum evaluation of the functional $M_{k+1}^{0, k}$ if the decomposition point is located inside the half-interval $[0, k)$.

3. If $M_{k+1}^{0, k}<M_{k+1}^{\text {current }}$, then $M_{k+1}^{0, k}$ and $[0, k)$ are sent to the priority queue.

4. While the priority queue is not empty

a. Takes from the priority queue the request $M_{k+1}^{b, e},[b, e)$ with the minimum value.

b.If $M_{k+1}^{b, e} \geq M_{k+1}^{\text {current }}$, the request is not processed and end of algorithm.

c.If $e-b<N$ (in our case $N=8$ )

then a loop: for $j$ from $b$ till $e-1$.

- Calculates $M_{k+1}^{j}$.

- If $M_{k+1}^{j}<M_{k+1}^{\text {current }}$, then $j^{\text {current }}=j, M_{k+1}^{\text {current }}=M_{k+1}^{j}$.

Else splits $[b, e)$ into two half-intervals $[b, j)$ and $[j, e)$.

- Calculates with expression (2) the minimum evaluations of the functionals $M_{k+1}^{b, j}$ and $M_{k+1}^{j, e}$, suggesting that the new decomposition point is located first in $[b, j)$ and then in $[j, e)$.

- If $M_{k+1}^{b, j}<M_{k+1}^{\text {current }}$, then $M_{k+1}^{b, j}$ and $[b, j)$ are sent to the priority queue.

- If $M_{k+1}^{j, e}<M_{k+1}^{\text {current }}$, then $M_{k+1}^{j, e}$ and $[j, e)$ are sent to priority queue.

At the end of the algorithm, $j^{*}=j^{\text {current }}$ and $M_{k+1}=M_{k+1}^{\text {current }}$.

Splitting a half-interval can be done in different ways. One of them is dividing by two. 